\title{
Tutorial de Pubmed Búsqueda en modo avanzado
}

\begin{abstract}
Búsqueda avanzada
En el modo avanzado de búsqueda usted puede especificar los campos donde se realizará la búsqueda, con lo cual puede construir una estrategia de búsqueda mediante la adición de términos (o conceptos) de a uno por vez. Además, usted puede modificar la estrategia de búsqueda mediante el uso de operadores booleanos (será abordado más adelante).
\end{abstract}

Es aconsejable que antes de compenetrarse con las características de la búsqueda avanzada, tenga conocimiento y práctica con el modo básico de búsqueda.

Para acceder al modo de búsqueda avanzada se debe hacer click en el hipervínculo "Advanced Search" de la barra de opciones (a la izquierda) en la página principal de PubMed. Una vez allí, nos encontramos con la interface de búsqueda avanzada como lo muestra la imagen:

En esta página observamos (los números se corresponden a las referencias de la imagen):

1) El menú desplegable del

"Search Fields" o campos de búsqueda. Luego ampliaremos su significado.

2) El "Mode search" menú (cabe destacar que el hipervínculo de los títulos de cada menú desplegable relaciona a la página de ayuda de cada uno repec-

\section{PubMed}
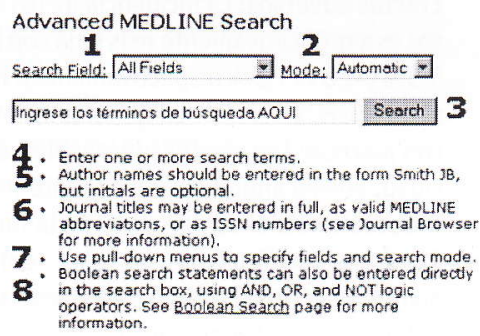
tivamente).

3) Inmediatamente por debajo se encuentra el casillero de texto en donde se ingresan los términos (keywords) de la búsqueda, junto al botón "Search" para desencadenar la misma.

Luego hay cinco instructivos (más adelante se amplia la información): 4) Ingrese uno o más términos de búsqueda (separar los términos por espacios).

5) Los nombres de autores de acuerdo al siguiente ejemplo: Perez JB (las iniciales son opcionales).

6) Los títulos de las publicaciones (Journals) pueden ingresarse completos (p.e., Journal of Nutrition), abreviados como los validados por MEDLINE (p.e., j nutr) o como números de ISSN (p.e., 0022-3166).

7) Use los menúes desplegables para especificar los campos y el modo de búsqueda.

8) Las expresiones de búsqueda booleana también pueden ser entradas directamente en el casillero de texto, usando los operadores lógicos AND, OR y NOT .

\section{Menú de campos de búsqueda:}

Cada cita de la base de datos del MEDLINE posee campos que informan sobre algún aspecto en particular de dicho artículo. Para realizar una búsqueda avanzada usando un campo de búsqueda específico, se puede seleccionar el campo desde el menú desplegable de "Search Field" o ingresar la expresión abreviada del campo (entre corchetes) en el casillero de texto, a continuación de cada término a buscar. Nótese que por defecto está seleccionada la opción "Todos los Campos" ("All Files").

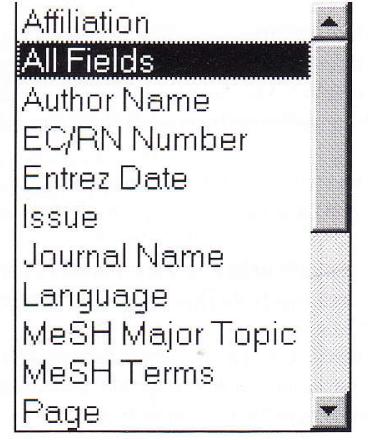

Estos campos poseen un nombre específico el cual es abreviado (recordar que se ingresan entre corchetes) según una convención. A continuación se describe sintéticamente cada uno de los incluidos en el menú:

Affiliation [AD] o [AFFL]: Adress of author. Contiene la afiliación institucional y la dirección del primer autor (algunas veces la de otros autores) al momento en que el artículo fue publicado. Este campo se encuentra disponible desde 1988, Las citas previas a esta fecha no lo contienen.

All Fields [ALL]: cubre todos los campos de búsqueda de la base de datos de PubMed (es la opción por defecto).

Author Name [AU] o [AUTH]: contiene la lista de los autores de cada artículo, se consignan hasta 10 autores, si son más de diez; el décimo es seguido de la expresión "et al".

E. C. Number [RN] o [ECNO]: es un número asignado por la "Enzyme Commission" para identificar a cada enzima en particular.

Entrez Date [ED]: la fecha de ingreso a la base Entrez que mantiene el PubMed.

Issue [ISSUE]: es el número del Journal en el cual el artículo fue publicado.

Journal Title [TA] o [JOUR]: es el nombre del Journal donde fue publicado el artículo. Estos están almacenados en su forma abreviada, pero pueden ser ingresados en la extensión completa, abreviada o el número de ISSN. Para conocer la lista de los Journals indizados es conveniente familiarizarse con el "Journal Browser".

Language [LA] o [LANG]: es el lenguaje en el cual el artículo fue publicado. Cabe destacar que muchos de los artículos que no fueron escritos originalmente en inglés aparecen con la expresión "non-English" a continuación del la lengua en que fue escrito el artículo. Dichos artículos poseen en la base de datos el abstract en inglés.

MeSH Major Topic [MAJR]: incluye todos los términos MeSH que fueron asignados como los más importantes para cada artículo por Los indizadores del MEDLINE de la National Library of Medicine.

MeSH Terms [MS] o [MESH]: incluye todos los términos del "Medical Subject Headings" (MeSH)", un diccionario de términos controlados utilizado para indicar el MEDLINE. Cada cita del MEDLINE tiene asignado un grupo de términos de este diccionario, los cuales se refieren al tema que versa dicho artículo.

Modification Date [MDAT]: contiene la fecha en la cual el artículo fue modificado en PubMed por última vez, en el formato año/mes/día.

Page Number [PAGE]: es el número de la primera página donde aparece publicado el artículo en el Journal.

Publication Date [DP] o [PDAT]: contiene la fecha en la que el artículo fue publicado, aparece en el formato año/mes/día. Un año solo (p.e. 1984) recuperaría todos los artículos publicados para ese año, un año y un mes (p.e. 1984/03) traería todos los de ese mes.

Publication Type [PT] o [PTYP]: se refiere a la forma de presentación de un artículo. Si desea puede consultar la lista de 
tipos de publicación. Por ejemplo: review articles, clinical trials, randomized controlled trials, retracted publications.

Substance [NM] o [SUBS]: contiene el nombre de cualquier asociación química entre el registro de "Chemical Abstract Service (CAS)" y el "MEDLINE Name of Substance field".

Text Words [TW] o [WORD]: incluye todas las palabras en el título y el abstract, más palabras individuales del MeSH y nombres de substancias químicas.

Title Words [TI] o [TITL]: incluye sólo aquellas palabras encontradas en el título de un registro.

Volume [VI] ó [VOL]: es el número del volúmen del Journal en el cual el artículo fue publicado.

Medline ID [UI] o [MUID]: es el identificador único que MEDLINE le asigna a cada registro de la base.

PubMed ID [PMID]: es el identificador único que PubMed le asigna a cada registro de la base.

Si se seleccionó algún campo en particular, o todos por defecto, el o los términos de nuestra estrategia de búsqueda serán procesados en los respectivos campos seleccionados, es importante recordar que en el modo avanzado de búsqueda también funcionan (salvo que se fuerce al motor de búsqueda de PubMed a no hacerlo) los mecanismos internos de búsqueda descriptos en el apartado del modo básico del presente tutorial.

\section{Menú de modo de búsqueda:}

Este menú desplegable de modo de búsqueda en PubMed le permite ingresar los términos de la búsqueda en dos modos:

\section{Automatic} List Terris:

Automática: es la opción por defecto, en la cual el o los términos que son ingresados son inmediatamente adicionados a la búsqueda. Si se ingresa más de una palabra, PubMed analizará la posibilidad de realizar la búsqueda como una frase; si no se encontrara una frase, automáticamente agregará el operador booleano AND a los términos para mostrar el resultado. Recordar que este es el mecanismo oculto que automáticamente se realiza en el modo básico de búsquedas.

Lista de términos: cada campo de término de búsqueda posee un índice de como está compuesto ese campo; por ejemplo para los tipos de publicación si son review, trials, etc.; para los términos MeSH los diferentes encabezamientos y subencabezamientos; para los journals la lista de los mismos; y así un índice para cada campo de búsqueda.

En el modo automático se busca solamente lo ingresado en el casillero de texto. En el modo de lista de términos se nos da la opción de navegar en los índices para acotar más la búsqueda. Tener en cuenta que de elegir está opción se pasa a una página intermedia previa a la de edición de consulta donde se puede seleccionar en una ventana el aspecto particular del campo de búsqueda en cuestión, pasando luego a la página de edición. En dicho índice si se desea seleccionar más de un término se debe orpimir la tecla "Ctrl-key" y marcar el término con el mouse. En el siguiente punto se amplía gráficamente esta importante característica de PubMed.

Seleccionando un término en el modo de búsqueda "automático": Para ejemplificar una búsqueda en Modo Avanzado nos pondremos como objetivo encontrar artículos que versen sobre prevención de tromboembolismo o varicela durante el embarazo (prevention of

the condition of thromboembolism or chikenpox during pregnancy).

En primer término seleccione el campo "MeSH Terms" desde el menú desplegable de campos de búsqueda, mantenga la opción "Automatic" en el menú Modo de búsqueda que permite el ingreso automático del término a buscar, en nuestro caso comenzaremos por pregnancy y luego oprima el botón "Search".

\section{PubMed QUERY}

Inmediatamente se mostrará una nueva página (PubMed.Query) en la cual se nos muestra el resultado de la búsqueda realizada, esta página es intermedia entre la interfase del Modo Avanzado de Búsqueda y la Página de Resultados. En está página intermedia es posible editar y trabajar con la estrategia de búsqueda (lo cual no era posible en el Modo Básico de Búsqueda). Esta página consta de tres partes:

1) Current Query: Se muestra la estrategia de búsqueda y la opción de recuperación junto a un botón "Details" que permite ver la estrategia en una nueva ventana.

\section{2) Add Term(s) to} Query: Opción con la que se pueden seguir incorporando términos a la estrategia de búsqueda. Cada término que se agregue a la estrategia será adicionado con el operador lógico AND.

3) Modify Current Query: Se puede modificar la actual estrategia de búsqueda.

Seleccionando un término en el modo de búsqueda "lista de términos" El hecho de poder navegar por los índices que conforman las diferentes listas de términos es, como se comentó en el apartado de como evaluar los servidores de Medline vía Web, uno de los aspectos que elevan el puntaje en dicha calificación, PubMed lo permite desde la opción de selección de un término en el modo de búsqueda "lista de términos" en el modo avanzado de búsqueda.

Más adelante veremos que para facilitar dicha búsqueda PubMed posee interfases especialmente diseñadas a tal fin, como por ejemplo el Journal Browser, el MeSH Browser, etc.

Continuando con nuestro ejemplo a continuación agregaremos el término MeSH Thromboembolism en la sección media de la página (Add Term(s) to Query) utilizando el modo de búsqueda List Terms, tal como lo muestra la imagen:

Add Term(s) to Query:

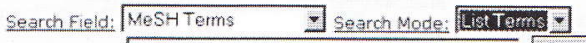

Enter Terms: Phromboembolism Clear

Enter one or more author last namss, toxt words, or other keywerds. To search for all terms that begin with a given word, place an asterisk (") of the end of the word. Journal Tities mus: be MED 
Luego de oprimir el botón Search nos dirigimos nuevamente a una página intermedia, en la cual ahora se nos muestra en una ventana con barra de desplazamiento una lista de términos, en nuestro caso del índice $\mathrm{MeSH}$, que coinciden con el término ingresado, a la derecha de los mismos entre paréntesis se indica la cantidad de citas indexadas en el MEDLINE con ese término MeSH.

Avatlable terms for the field MeSH Terms (Number of Records)

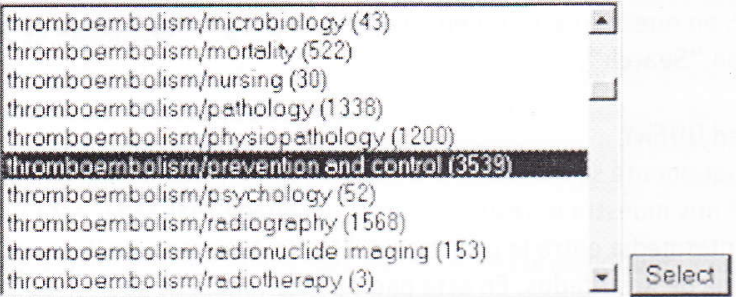

Ahora recorra dicha ventana y seleccione prevención de tromboembolismo (thromboembolism/prevention and control) observe que los subencabezamientos (subheadings) están a continuación de cada término MeSH luego de la barra /

Recuerde que si desea seleccionar más de un término se debe oprimir la tecla "Ctrl" y marcar el término con el mouse. Posteriormente debe oprimir el botón "Select" con lo cual nos dirigimos a la página de formulaciôn de la estrategia avanzada de búsqueda o "PubMed QUERY" ya comentada.

\section{Current Query}

Una vez que se adicionó el segundo término a la estrategia de búsqueda, podemos en la primera sección de la pantalla del PubMed Query, la llamada Current Query o Consulta Actual, recuperar los artículos que tienen los términos requeridos en nuestra consulta (Ref. 1), también nos muestra la descripción de la estrategia (Ref. 2) en este caso los dos términos MeSH unidos por el operador booleano AND.

HCDI PubMed PubMed QUERY: PubMed?

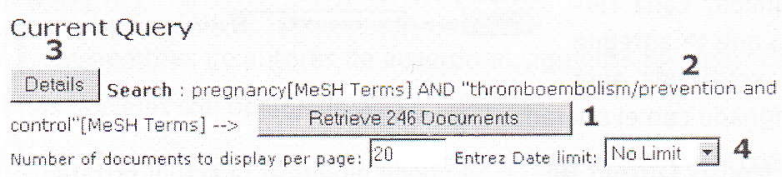

Si deseáramos ver en detalle la formulación de la estrategia (Ref. 3) oprimiendo el botón "Details" se abriría una nueva ventana con dicho detalle. Es posible también limitar en cantidad de documentos por página y fecha de publicación de las citas tal como lo viéramos en el Modo Básico (Ref. 4).

Hasta aquí obtuvimos 246 citas sobre prevención de tromboembolismo en embarazo, debemos seguir adicionando términos a nuestra consulta por lo cual nuevamente nos dirigiremos a la sección media de la pantalla. Term (s) to Query:

En Add Term(s) to Query se pueden ingresar más términos a la estrategia de búsqueda, mantendremos el modo de List Terms e ingresamos chikenpox (varicela)

Luego de oprimir el botón Search, nuevamente pasaríamos a una página intermedia para la selección del término, como fue explicado anteriormente, y se podría seguir asi sucesivamente hasta quedar conforme con la estrategia de búsqueda.

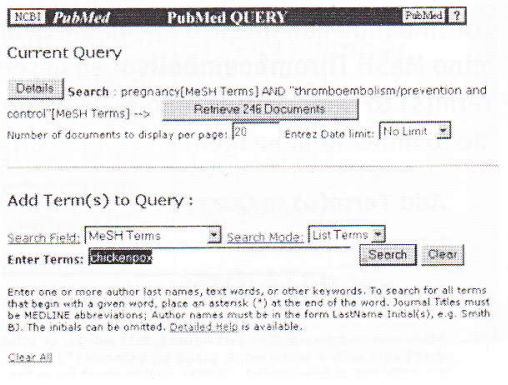

En nuestro caso seleccionamos chikenpox/prevention and control.

Luego de opri-

mir el botón

Select, vemos

en la sección

Current Query

que NO se po-

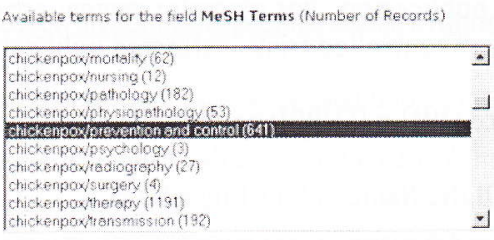

$v$ Select

drá recuperar ninguna cita, esto es porque automáticamente se agregó el término varicela con el operador AND, con lo cual no hay ningún artículo que verse sobre prevención de tromboembolismo y varicela en el embarazo, por lo cual debemos cambiar el operador lógico modificando la consulta actual.

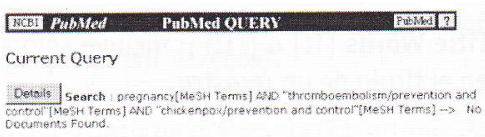

\section{Modify Current Query}

PubMed combina sus términos de búsqueda con el operador booleano AND, el cual puede ser modificado a pie de página en la última de las opciones del PubMed QUERY, dicha opción, el Modify Current Query, permite modificar la estrategia formulada, con el uso de operadores booleanos, tal como lo muestra la imagen en el menú desplegable.

Para seleccionar los términos se debe oprimir la tecla "Ctrl-key" y marcar con el mouse el término. Las opciones que se presentan en el menú desplegable son:

\section{Modify Current Query:}

Term (Total Records)

\begin{tabular}{|c|c|c|}
\hline \multicolumn{3}{|c|}{$\begin{array}{l}\text { pregnancy (MeSHTerms) (409724) } \\
\text { "pregnoncy [MeSH Tems] (409724) } \\
\text { thromboembolismMeSH Terms)(20345) } \\
\text { "thromboembolismprewention and control"(MeSH Tems] (3539) } \\
\text { chickenpox(MeSH Terms] (3541) } \\
\text { "chickenpoxprevention and control'[MeSH Terms] (641) }\end{array}$} \\
\hline \multirow[t]{2}{*}{ Search } & Intersection (AND) & \multirow{2}{*}{ of the selected term } \\
\hline & Intersection (AND) & \\
\hline Questions or comr & $\begin{array}{l}\text { Oifterence (BUTNoT) } \\
\text { Penge }\end{array}$ & Losk. \\
\hline
\end{tabular}

Intersección (AND): solo aquellas citas que contienen todos los términos especificados.

Unión (OR): citas que contienen cualquiera de los términos especificados. Diferencia (NOT): excluye las citas con los términos especificados. Range: rango de los términos especificados (p.e. Publication Date)

Siguiendo nuestro ejemplo debemos modificar la búsqueda ya que el operador booleano AND no era el que necesitabamos, ahora seleccione los términos MeSH thromboembolism/prevention and control y mante-

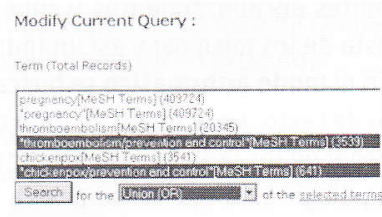
niendo la tecla "Ctrl" presionada, con el puntero del mouse seleecione chikenpox/prevention and control, Luego elija del menú deplegable el operador booleano OR de unión y corra nuevamente la búsqueda oprimiendo el botón "Search".

Una vez que se unieron los dos términos MeSH con el operador OR debemos nuevamente unirle con el AND el término embarazo para que finalmente quede la estra- modtrcurentener. tegia que formulamos inicialmente "prevención de tromboembolismo o varicela durante el embarazo"

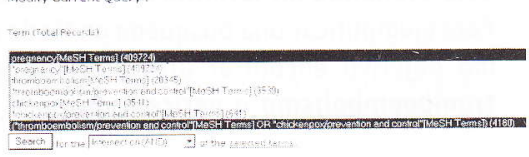


Por último al unir ambos términos con el operador AND (el que está por defecto) vemos como queda la estrategia finalmente:

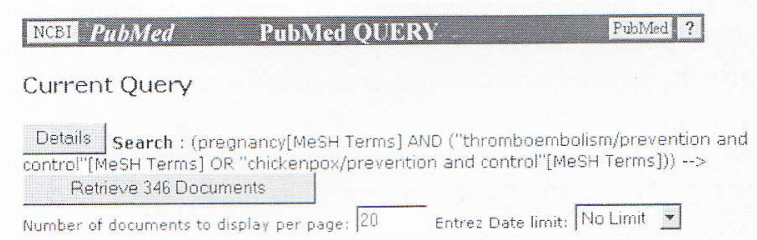

Al oprimir el botón de recuperación, pasaríamos a la página de resultados ya descripta previamente, donde veremos (según las limitaciones que se asignen para dicha página) la cantidad de citas estipulada en la consulta actual.

\section{Mce! PabNed PubMed OULRY Publad? \\ Details (pregnoncy[MeSHTerms] AND (thromboembo Search Clear \\ Docs Per page: 20 IEntrez Date limit: Nolimit - \\ citations 1-20 displayed (out of 346 found), page 1 of 18

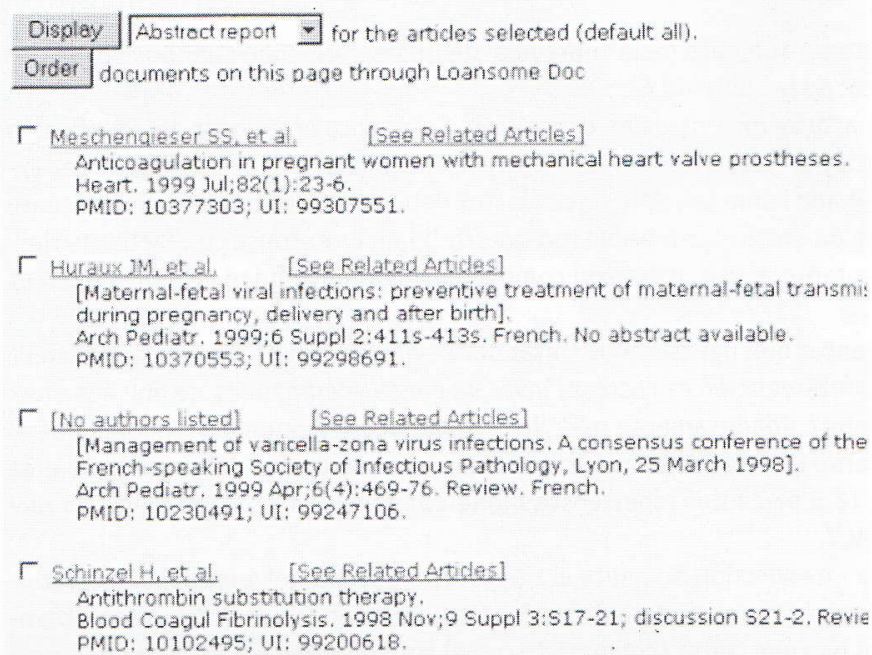

Ejercicio práctico

Realizando una búsqueda en modo avanzado:

Ya en la página de Búsqueda Avanzada, como ejemplo intentaremos buscar artículos que versen sobre:

Etiología de cáncer y enfermedades de transmisión sexual como punto principal del artículo, excluyendo infecciones por HIV, en idioma inglés desde 1992 hasta 1993.

En primer lugar desplegaremos el menú de campos de búsqueda y elegiremos el campo "MeSH Major Topic" (podría elegir el que más nos interese o por defecto dejar todos los campos).

En el casillero de texto ingresaremos el término sexually transmitted diseases (recordar que se podría poner en el casillero de texto el término más la abreviación del campo entre corchetes: sexually transmitted diseases [MAJR], siendo esto lo mismo).

Observe que por defecto el modo de búsqueda en este caso fue el automático. Ahora oprimimos el botón "Search".
Pasamos a una pácina para continuar editando nuestra búsqueda, en la parte intermedia de la pantalla encontramos la posibilidad de agregar nuevos términos a la búsqueda antes de pedir la recuperación de los documentos. Es la misma interface que la página inicial del modo avanzado. Seleccionamos el campo de búsqueda "MeSH Major Topic" nuevamente e ingresamos en el casillero de texto neoplasms, ya que lo que realmente necesitamos es etiología pero como no conocemos el subencabezamiento del MeSH para etiología, seleccionaremos el modo de "Term List" para navegar por dicho índice y elegir el que corresponda, hecho esto luego oprimimos "Search".

Ahora pasamos a una página intermedia en donde se encuentra el índice de los términos MeSH, seleccionamos neoplasms/etiology (observe que a la derecha entre paréntesis aparece la cantidad de citas que existen bajo este término en particular) y luego oprimimos "Select".

Nos encontramos ahora nuevamente en la página de edición de la consulta con la posibilidad de agregar más terminos si lo deseamos o acotar la forma de presentación de los documentos en cantidad por página o año de publicación. Cabe señalar que en definitiva se está realizando una búsqueda booleana y debido a que la lectura de una estrategia de búsqueda de este tipo es de izquierda a derecha, se debe ir encerrando en paréntesis los grupos de inclusión (en este caso neoplasms/etiology y sexually transmitted diseases) fuera de los de exclusión (HIV infections), por lo cual al final de la página aparece el "Modify Current Query" con lo cual selecciono sexually transmitted diseases con el cursor del mouse, luego oprimiendo la tecla Ctrl-key selecciono neoplasms/etiology y con el operador lógico AND oprimo el botón "Search". De esta manera encerré entre paréntesis ambos términos (vea que aparece así en el cuadro de texto del "Modify Current Query").

Ahora selecciono "MeSH Term" como campo de búsqueda en modo automático, e ingreso HIV infections en el cuadro de texto de "Modify Current Query". Si en este punto en la primera parte de la página le aparece la fórmula de búsqueda de la siguiente manera:

(("Sexually Transmitted Diseases" [MeSH Major Topic] AND "neoplasms/etiology"[MeSH Major Topic]) AND HIV infections[MeSH Terms]) va por buen camino, de no ser así, inténtelo nuevamente. Observe que lo que se desea es excluir como causa las infecciones por HIV por lo cual debe dirigirse al final de la página y seleccionar ("Sexually Transmitted Diseases"[MeSH Major Topic] AND "neoplasms/etiology"[MeSH Major Topic]) y luego HIV infections[MeSH Terms] posteriormente marque el operador lógico BUTNOT y oprima el botón "Search".

Ahora tendría que aparecer: (("Sexually Transmitted Diseases"[MeSH Major Topic] AND "neoplasms/etiology"[MeSH Major Topic])) BUTNOT (HIV infections[MeSH Terms])

Por último para acotar a un rango determinado de tiempo seleccionar "Publication Date"en campos de búsqueda y en modo automático, ingresar 1992:1993 (observe los dos puntos entre los años).

De esta manera terminamos con nuestra estrategia, si la misma quedó conformada así: ((( ("Sexually Transmitted Diseases" [MeSH Major Topic] AND "neoplasms/etiology" [MeSH Major Topic]) NOT HIV infections [MeSHTerms]) ANDenglish [Language]) AND 1992:1993 [Publication Date])

con alrededor de 60 artículos para recuperar, TUVO EXITO!!!!! 